BULLETIN OF THE

AMERICAN MATHEMATICAL SOCIETY

Volume 77, Number 4, July 1971

\title{
DUALITY AND VON NEUMANN ALGEBRAS ${ }^{1}$
}

\author{
BY MASAMICHI TAKESAKI
}

Communicated by Ray A. Kunze, February 19, 1971

The aim of this paper is to announce the results of the author's lecture given in Tulane University for the Fall of 1970 under the same title. Since the Pontryagin duality theorem was shown, a series of duality theorems for nonabelian groups has been discovered, Tannaka duality theorem [14], Stinespring duality theorem [10], Eymard-Saito duality theorem [5], [8] and Tatsuuma duality theorem [15]. Motivated by the Stinespring duality theorem, Kac [7] introduced the notion of "ring-groups" in order to clarify the duality principle for unimodular locally compact groups. Sharpening and generalizing Kac's postulate for the "ring-group," the author [11] gave a characterization of the group algebra of a general locally compact group as an involutive abelian Hopf-von Neumann algebra with left invariant measure.

Let $G$ be a locally compact group with left Haar measure $d s$. Let $\mathfrak{S}$ denote the Hilbert space $L^{2}(G, d s)$. Define a unitary operator $W$ on $\mathfrak{S} \otimes \mathfrak{S}$ by $(W f)(s, t)=f(s, s t), f \in \mathfrak{S} \otimes \mathfrak{S}, s, t \in G$. Let $\mathbb{Q}(G)$ be the von Neumann algebra on $\mathfrak{S}$ consisting of all multiplication operators $\rho(f)$ by $f \in L^{\infty}(G)$. The algebras $Q(G)$ and $L^{\infty}(G)$ will be identified. Let $\operatorname{Tr}(G)$ denote the von Neumann algebra on $\mathfrak{S}$ generated by left regular representation $\lambda$ of $G$. The fundamental facts of all duality arguments for groups are the following: the map $\delta_{G}: x \mapsto W(x \otimes 1) W^{*}$ is an isomorphism of $Q(G)$ into $Q(G) \bar{\otimes} Q(G)$ such that $\left(\delta_{G} \otimes i\right) \circ \delta_{G}=\left(i \otimes \delta_{G}\right) \circ \delta_{G}$; the map $\gamma_{G}: x \mapsto W^{*}(x \otimes 1) W$ is an isomorphism of $\mathfrak{N (}(G)$ into $\mathfrak{N}(G) \bar{\otimes} \mathfrak{\Re ( G )}$ such that $\left(\gamma_{G} \otimes i\right) \circ \gamma_{G}$ $=\left(i \otimes \gamma_{G}\right) \circ \gamma_{G}$ and $\sigma \circ \gamma_{G}=\gamma_{G}$ where $\sigma$ denotes the automorphism of $B(\mathfrak{S}) \bar{\otimes} B(\mathfrak{S})$ defined by $\sigma(x \otimes y)=y \otimes x$. According to these facts, the preduals $\mathfrak{Q}_{*}(G)$ and $\mathfrak{T H}_{*}(G)$ of $Q(G)$ and $\mathfrak{T}(G)$ turn out to be Banach algebras by the following multiplications: $\langle x, \psi * \varphi\rangle$ $=\langle\delta(x), \psi \otimes \varphi\rangle, x \in Q(G), \psi, \varphi \in Q_{*}(G)$ and $\langle x, \varphi * \psi\rangle=\langle\gamma(x), \varphi \otimes \psi\rangle$, $x \in \operatorname{Mr}(G), \varphi, \psi \in \mathfrak{T N}_{*}(G)$. The Banach algebra $\mathfrak{Q}_{*}(G)$ is nothing but the usual group algebra $L^{1}(G)$ and the duality theorems mention that the Banach algebra $\mathfrak{I}_{*}(G)$ is semisimple and the spectrum space

AMS 1970 subject classifications. Primary 46L10, 22D35, 22 D25.

Key words and phrases. Duality, locally compact groups, operator algebras, Hopfvon Neumann algebras, regular representation, representations, Peter-Weyl theorem.

1 The preparation of this paper was supported in part by NSF Grant \#GP-18127. 
of $\mathfrak{T}_{*}(G)$ is the given group $G$ itself by the map: $\varphi \in \mathfrak{N}_{*}(G) \mapsto\left\langle\lambda(s)^{*}, \varphi\right\rangle$, $s \in G$.

Noticing this scheme of duality principle for groups, we develop the duality theory for more general subjects, Hopf-von Neumann algebras, based on the theory of von Neumann algebras. A Hopf-von Neumann algebra is a pair $\{a, \delta\}$ of a von Neumann algebra $Q$ and a normal isomorphism $\delta$ of $Q$ into $Q \bar{\otimes} Q$ such that $(\delta \otimes i) \circ \delta=(i \otimes \delta)$ $\circ \delta$, where $i$ denotes the identity automorphism of $\propto$. The isomorphism $\delta$ is called a comultiplication of $Q$. If $Q$ is abelian, then $\{Q, \delta\}$ is said to be abelian. If $\{a, \delta\}$ is a Hopf-von Neumann algebra, then the predual $a_{*}$ of $a$ turns out to be a Banach algebra with the product, called the convolution, given by $\langle x, \varphi * \psi\rangle=\langle\delta(x), \varphi \otimes \psi\rangle$, $x \in Q, \varphi, \psi \in Q_{*}$. In the tensor product $Q \bar{\otimes} Q$, let $\sigma$ denote the automorphism defined by $\sigma(x \otimes y)=y \otimes x, x, y \in Q$. If $\sigma \circ \delta=\delta$, then $\delta$ is said to be symmetric. The resulted Banach algebra $Q_{*}$ is abelian if and only if $\delta$ is symmetric. The involution of $\{Q, \delta\}$ is an anti-automorphism $\nu$ of $Q$, such that $\nu^{2}=i$ and $(\nu \otimes \nu) \circ \delta=\sigma \circ \delta \circ \nu$. The triplet $\{a, \delta, \nu\}$ is called an involutive Hopf-von Neumann algebra. In this case, the Banach algebra $Q_{*}$ admits the involution: $\varphi \in Q_{*} \mapsto \varphi^{\#} \in Q_{*}$ defined by $\left\langle x, \varphi^{\#}\right\rangle=\left\langle\nu\left(x^{*}\right), \varphi\right\rangle^{-}, x \in Q, \varphi \in Q_{*}$. In the following, we write $\nu(a)=a^{\iota}, \nu\left(a^{*}\right)=a^{\mathrm{b}}$ for $a \in Q$ and ${ }^{t} \nu(\varphi)=\varphi^{\wedge}$ for $\varphi \in Q_{*}$. If a faithful, semifinite, normal trace $\tau$ of $\propto$ satisfies the equation:

$$
(\tau \otimes \tau)((a \otimes b) \delta(c))=(\tau \otimes \tau)\left(\left(a^{\wedge} \otimes c\right) \delta(b)\right)
$$

for every $a, b$ and $c$ in $L^{1}(\tau) \cap a$, then $\tau$ is called a left invariant measure on $\{a, \delta, \nu\}$. A right invariant measure is also defined in the similar way. If $\nu$ leaves $\tau$ invariant, then $\tau$ is said to be unimodular.

For a locally compact group $G, Q(G)$ and $\operatorname{TC}(G)$ both admit the comultiplications $\delta_{G}$ and $\gamma_{G}$ respectively. The involutions $\nu_{G}$ of $\left\{Q(G), \delta_{G}\right\}$ and $\kappa_{G}$ of $\left\{\operatorname{TT}(G), \gamma_{G}\right\}$ are defined respectively by $\nu_{G}(f)(s)=f\left(s^{-1}\right), f \in Q(G), s \in G$, and $\kappa_{G}(x)=C x^{*} C, x \in \mathscr{T}(G)$, where $C$ denotes the conjugation $C: \xi \in \mathfrak{S} \mapsto \xi \in \mathfrak{S}$. The trace $\mu_{G}$ on $Q(G)$ defined by $\mu(f)=\int_{G} f(s) d s$ is indeed a left invariant measure of $\left\{a(G), \delta_{G}, \nu_{G}\right\}$. If $G$ is unimodular, then the canonical measure $\varphi_{G}$ defined on $\operatorname{Tr}(G)$ is a unimodular measure of $\left\{\mathfrak{M}(G), \gamma_{G}, \kappa_{G}\right\}$.

Suppose now an involutive Hopf-von Neumann algebra $\{a, \delta, \nu, \tau\}$ with left invariant measure is given.

LEMMA 1. There exists an ideal $\mathfrak{a}$ of a contained in the definition ideal $\mathfrak{m}_{\tau}$ of the trace $\tau$ such that

(i) $\mathfrak{a}$ is invariant under both $\nu$ and the transpose ${ }^{t} \nu$ of $\nu$;

(ii) $\mathfrak{a}$ is dense in the Hilbert space $L^{2}(a, \tau)$; 
(iii) the map: $a \in \mathfrak{a} \mapsto^{t} \nu \circ \nu(a) \in \mathfrak{a}$ is essentially selfadjoint in $L^{2}(a, \tau)$ and the closure $\Delta$ of this linear map is positive and nonsingular;

(iv) $a$ is closed under the convolution product.

Regarding $Q$ as a function system, we denote its elements by $f, \quad g, \cdots$. A linear map: $\sum_{i=1}^{n} f_{i} \otimes g_{i} \in \mathfrak{a} \otimes \mathfrak{a} \mapsto \sum_{i=1}^{n} \delta\left(g_{i}\right)\left(f_{i} \otimes 1\right)$ $\in Q \otimes Q$ is extended to a unitary operator $W$ on $L^{2}(Q \bar{\otimes} Q, \tau \otimes \tau)$. Representing $a$ on $L^{2}(\tau)$, we have $\delta(a)=W(1 \otimes a) W^{*}, a \in a$. The convolution product and the involution: $f \mapsto f^{\#}$ makes $\mathfrak{a}$ into a left (generalized) Hilbert algebra in the sense of [12]. Let $\lambda(f), f \in \mathfrak{a}$, denote the convolution operator: $g \mapsto f * g$. Then this map $\lambda: f \in \mathfrak{a} \mapsto \lambda(f)$ is extended uniquely to a ${ }^{*}$-representation of $a_{*}=L^{1}(\tau)$, which is also denoted by $\lambda$. Let $\mathfrak{M}(\lambda)$ denote the von Neumann algebra generated by $\lambda(f), f \in L^{1}(\tau)$. Then $\mathfrak{T}(\lambda)$ is nothing but the left von Neumann algebra of the left Hilbert algebra. Using the selfadjoint unitary operator $V: f \mapsto \Delta^{-1 / 2} f^{2}$, we can define another representation $\lambda^{\prime}: f$ $\in L^{1}(\tau) \mapsto V \lambda(f) V$, which is called the right regular representation of $L^{1}(\tau)$. The original one $\lambda$ is called the left regular representation. Let $\operatorname{Tr}\left(\lambda^{\prime}\right)$ denote the von Neumann algebra generated by $\lambda^{\prime}(f), f \in L^{1}(\tau)$. Then we have

THEOREM 1. $\mathfrak{T}(\lambda)^{\prime}=\mathfrak{T}\left(\lambda^{\prime}\right)$.

Theorem 2. $W \in Q \bar{\otimes} \mathfrak{M}(\lambda)$.

THEOREM 3. The map $\gamma: x \mapsto \sigma\left(W^{*}(x \otimes 1) W\right)$ is a comultiplication of $\operatorname{Tr}(\lambda)$.

Let $C$ denote the conjugation: $f \in L^{2}(\tau) \mapsto f^{*} \in L^{2}(\tau)$. Then the map $\kappa: x \mapsto C x^{*} C$ is an involution of the Hopf-von Neumann algebra $\{\mathfrak{M}(\lambda), \gamma\}$.

The canonical weight $\varphi$ of $\mathfrak{T}(\lambda)$ given by the left Hilbert algebra $\mathfrak{a}$, see [1], [2] and [13], behaves as if it were a unimodular measure on $\{\operatorname{Tr}(\lambda), \gamma, \kappa\}$. Based on $\{\operatorname{Tr}(\lambda), \gamma, \kappa, \varphi\}$, we can construct a Hilbert space $L^{2}(\varphi)$, the left regular representation $\rho_{0}$ of the involutive Banach algebra $\mathfrak{T}_{*}(\lambda)$ with convolution product, a comultiplication $\delta_{0}$ of the von Neumann algebra $\operatorname{Mr}\left(\rho_{0}\right)$ generated by $\rho_{0}(\varphi), \varphi \in \mathscr{M}_{*}(\lambda)$, an involution $\nu_{0}$ of the Hopf-von Neumann algebra $\left\{\mathscr{T}\left(\rho_{0}\right), \delta_{0}\right\}$ and the canonical trace $\tau_{0}$ on $\mathfrak{T}\left(\rho_{0}\right)$. Then we get

TheOREM 4 (DUALITY). There exists a unitary operator $\Lambda$ of $L^{2}(\tau)$ onto $L^{2}(\varphi)$ which sets up an isomorphism of $\{a, \delta, \nu, \tau\}$ onto $\left\{\operatorname{TT}\left(\rho_{0}\right), \delta_{0}, \nu_{0}, \tau_{0}\right\}$.

Theorem 5. The following statements (Ci) and (Cii) (resp. (Di) and (Dii)) are equivalent: 
(Ci) The trace $\tau$ is finite;

(Cii) the convolution algebra $\mathfrak{T}_{*}(\lambda)$ admits an identity.

(Di) The canonical weight $\varphi$ of $\mathfrak{T}(\lambda)$ is finite;

(Dii) the convolution algebra $L^{1}(\tau)=\mathfrak{Q}_{*}$ admits an identity.

Either (Ci) or (Cii) implies the unimodularity of $\tau$.

If either ( $\mathrm{Ci}$ ) or ( $\mathrm{Cii})$ holds, then $\{a, \delta, \nu, \tau\}$ is called compact. On the contrary, if either (Di) or (Dii) holds, then $\{a, \delta, \nu, \tau\}$ is said to be discrete.

Theorem 6 (Peter-WeyL). (i) If $\{\propto, \delta, \nu, \tau\}$ is compact, then the left regular representation $\lambda$ of $L^{1}(\tau)$ is decomposed into the direct sum of finite-dimensional irreducible representations with finite multiplicity; hence the von Neumann algebra $\operatorname{Tr}(\lambda)$ is atomic and finite, so of type I. Furthermore, every irreducible representation of $L^{1}(\tau)$ is finite-dimensional and unitary equivalent to a component of $\lambda$.

(ii) If $\{a, \delta, \nu, \tau\}$ is discrete, then the dual statement of (i) for $\mathfrak{T}_{*}(\lambda)$ holds.

THEOREM 7. (i) If $\{a, \delta, \nu, \tau\}$ is abelian, then there exists uniquely a locally compact group $G$ such that $\left\{\propto(G), \delta_{G}, \nu_{G}, \tau_{G}\right\}$ is isomorphic to $\{a, \delta, \nu, \tau\}$.

(ii) If $\{a, \delta, \nu, \tau\}$ is symmetric, then there exists uniquely a unimodular locally compact group $G$ such that $\left\{\mathfrak{T}(G), \gamma_{G}, \kappa_{G}, \varphi_{G}\right\}$ is isomorphic to $\{a, \delta, \nu, \tau\}$.

Applying our theory, we can get the Pontryagin, Tannaka, Stinespring, Eymard-Saito, Tatsuuma duality theorems for locally compact groups.

The whole theory announced above will be published as Tulane University Lecture Notes.

Acknowledgment. The author expresses his thanks to the Department of Mathematics, Tulane University, for their invitation which enabled him to develop this theory.

\section{REFERENCES}

1. F. Combes, Poids sur une $C^{*}$-algèbre, J. Math. Pures Appl. (9) 47 (1968), 57100. MR 38 \#5016.

2. —- Poids associê à une algèbre hilbertienne a gauche (to appear).

3. J. Dixmier, Les $C^{*}$-algèbres et leurs représentations, 2ième éd., Cahiers Scientifiques, fasc. 29, Gauthier-Villars, Paris, 1969. MR 39 \#7442.

4. J. Ernest, Hopf-von Neumann algebras, Proc. Conf. Functional Analysis (Irvine, Calif., 1966), Academic Press, London; Thompson, Washington, D.C., 1967, pp. 195-215. MR 36 \#6956.

5. P. Eymard, L'algèbre de Fourier d'un groupe localement compact, Bull. Soc. Math. France 92 (1964), 181-236. MR 37 \#4208. 
6. K. H. Hofmann, The duality of compact semigroups, and $C^{*}$-bigebras, Lecture Notes Series, no. 129, Springer-Verlag, Berlin and New York, 1970.

7. G. I. Kac, Ring groups and the duality principle, Trudy Moskov. Mat. Obšc. 12 (1963), 259-301 = Trans. Moscow Math. Soc. 1963, 291-339. MR 28 \#164; II, Trudy Moskov. Mat. Obšč. 13 (1965), 84-113=Trans. Moscow Math. Soc. 1965, 94-126. MR 33 \#226.

8. K. Saito, On a duality for locally compact groups, Tôhoku Math. J. (2) 20 (1968), 355-367. MR 39 \#357.

9. I. E. Segal, A non-commutative extension of abstract integration, Ann. of Math. (2) 57 (1953), 401-457; correction, ibid. (2) 58 (1953), 595-596. MR 14, 991; MR 15,204 .

10. W. F. Stinespring, Integration theorems for gages and duality for unimodular groups, Trans. Amer. Math. Soc. 90 (1959), 15-56. MR 21 \#1547.

11. M. Takesaki, A characterization of group algebras as a converse of TannakaStinespring-Tatsuuma duality theorem, Amer. J. Math. 91 (1969), 529-564. MR 39 \#5752.

12. - - Tomita's theory for modular Hilbert algebras and its applications, Lecture Notes Series, no. 128, Springer-Verlag, Berlin and New York, 1970.

13. - The theory of operator algebras, Lecture Notes UCLA, Los Angeles, Calif., 1969/70.

14. T. Tannaka, Über den Dualität der nichtkommutativen topologischen Gruppen, Tôhoku Math. J. 45 (1938), 1-12.

15. N. Tatsuuma, A duality theorem for locally compact groups, J. Math. Kyoto Univ. 6 (1967), 187-293. MR 36 \#313,

16. M. Tomita, Standard forms of von Neumann algebras, The Fifth Functional Analysis Sympos., Math. Soc. Japan, Sendai, 1967.

17. M. E. Walter, Group duality and isomorphisms of Fourier and Fourier-Stieltjes algebras from a $W^{*}$-algebra point of view, Bull. Amer. Math. Soc. 76 (1970), 1321-1325.

University of California, Los Angeles, California 90024 